\title{
Synthesis of sinapine and its unprecedented ruthenium-catalyzed $[2+2]$ photodimerization
}

\author{
Josephine M. Gießel, Tim Kohlmann, Tina Zeitz, Ralph Kluge and René Csuk* \\ Full Address: Martin-Luther-University Halle-Wittenberg, Organic Chemistry, Kurt-Mothes-Str. 2, D-06120 \\ Halle (Saale), Germany
}

\begin{abstract}
Sinapine was easily synthesized from commercially available starting materials in an overall yield of $32 \%$ while $0.47 \%$ were obtained by its extraction from white mustard. Irradiation of $\mathbf{1}$ in the presence of $\mathrm{Ru}(\mathrm{dndp})_{3} \mathrm{Cl}_{2}$ furnished in a [2+2] cycloaddition cyclobutanoid dimer, a $\delta$-truxinic acid. The putative mechanism of this reaction was supported by DFT calculations.
\end{abstract}

Keywords: sinapic acid, sinapine, dimerization, photoinduced, truxinic acid, ruthenium catalyst

\section{Introduction}

Secondary natural products have been of great importance to humankind for thousands of years ${ }^{1}$. Whereas in ancient times extracts from different plants were used to treat or alleviate diseases based on empirical observations, today these compounds have become indispensable as leads for the development of selective and most important drugs ${ }^{2-3}$. Refined analytical methods allow the discovery of highly active compounds that occur only in small amounts in plants, and advances in synthesis ensure the accessibility of these substances.

Thus, cyclobutanes have been isolated from many natural products starting with the isolation of $\alpha$ - and $\beta$-pinenes and caryophyllene by $\mathrm{O}$. Wallach in the 1890 er years ${ }^{4}$. Although these strained mono-cyclic rings were regarded in the beginning to be somewhat exotic; they could be found and isolated in many plants but only in a very tiny amount, but curiosity in these molecules together with the better isolation techniques and the development of synthetic strategies enable access to many of them. This development was driven in particular by the fact that some cyclobutanoid dimers ${ }^{5-10}$, such as innovanoside A, showed high biological activity. Furthermore, numerous cyclobutanoids of superior biological activity were isolated from the Piper genus, such as dipiperamides A-E ${ }^{11-18}$ and piperarborenines A-E ${ }^{19-28}$ (Scheme 1) and several total syntheses have been developed. However, there are only a limited number of synthetic approaches to these strained semi-dimers and homo-dimers, such as cross- coupling, re-arrangement, $\mathrm{C}$-H-functionalization and cycloaddition reactions.

A couple of years ago, piperlongumine (isolated from Piper longum ${ }^{29-31}$ has been found to exhibit superior cytotoxic properties. Recently, we were able to access several dimers of piperlongumine ${ }^{32-33}$, and to show them of even higher cytotoxicity than the parent compound.

These dimers were obtained by solid-state photodimerization. Photochemical transformations are of increasing importance for the synthesis of natural products as unique structures can be accessed otherwise being challenging to synthesize, and products can be obtained through high energy intermediates that remain unreachable by thermaldriven reactions ${ }^{34}$. As a consequence, in the last decades, the importance of light-induced reactions increased, and the introduction of LEDs presented a new state-of-the-art in science and technology ${ }^{35}$.

Recently, we became interested in sinapine ( 1 as its thiocyanate, Scheme 1) ${ }^{36-37}$, an alkaloid which can be found in white mustard (Sinapis alba) ${ }^{38-39}$ and canola (Brassica sp.) ${ }^{40-41}$ because some interesting biological activities have been reported for this hybrid composed from (E) 4-hydroxy-3,5dimethoxycinnamic acid and choline. Among other activities, this molecule holds anti-inflammatoric ${ }^{42-43}$, anti-oxidative ${ }^{44}$ and anti-angiogenic ${ }^{29-30}$ properties, as well as some potential as an inhibitor for the enzyme acetylcholinesterase ${ }^{45}$. Shown as early in $1852^{46}$, it can be extracted from plant material, and 
there is also one lengthy synthesis reported by E. Späth in $1920^{47}$.

\section{Results and Discussion}

To investigate the cytotoxicity as well as other biological activities of sinapine dimers, it was necessary to obtain larger amounts by synthesis. For comparison, authentic sinapine was obtained by extraction from white mustard (obtained from a local supermarket) using $n$-hexane and methanol followed by precipitation with an aqueous solution of KSCN gave sinapine as its thiocyanate (1) as a colorless solid in an overall yield of $0.47 \%$.

For a synthesis of sinapine thiocyanate, 4-hydroxy3,5-dimethoxy-benzaldehyde (2) was silylated to yield $94 \%$ of $\mathbf{3}$, whose Wittig olefination with carbomethoxymethyl triphenylphosphonium bromide/sodium hydroxide furnished $\mathbf{4}$ in $88 \%$ isolated yield. Compound $\mathbf{4}$ was hydrolysed with sodium hydroxide in ethanol (yielding 5) and finally allowed to react with $N, N, N$-trimethyl- $N$-(2chloroethyl)-ammonium chloride ( 7 that has been prepared from choline chloride (6) with thionyl chloride in $76.9 \%$ yield) followed by precipitation with $\mathrm{KSCN}$ in $46 \%$ yield. Thus, the overall yield of $32 \%$ was achieved. The material from the synthesis was identical to the material obtained by the extraction of the white mustard seeds (m.p., m.m.p, NMR, IR, MS).

Previously, the irradiation of piperlongumine, on a silica gel TLC plate with UV light $\lambda=254 \mathrm{~nm}$ was reported to afforded dimerization products 32 . Interestingly, under the same conditions, the irradiation of $\mathbf{4}$ for $2 \mathrm{~h}$ led to the exclusive formation of $\mathbf{8}$. Compound $\mathbf{8}$ is the corresponding (Z) stereoisomer of the starting material. In the ${ }^{1} \mathrm{H}$ NMR spectrum for $\mathbf{8}$ a vicinal coupling constant for the protons of the olefinic bond ${ }^{3} J=12.9 \mathrm{~Hz}$ was determined while for parent $4^{3} \mathrm{~J}=15.9 \mathrm{~Hz}$ was found, the latter being typical for a trans-configurated double bond. Hydrolysis of $\mathbf{8}$ gave acid $\mathbf{9}$.

Several drawbacks are found with large-scale photodimerization reactions using standard UV lamps, such as the development of heat and the high-energy consumption of the lamps. However, a couple of years ago, a [2+2] cycloaddition of acyclic enones using visible light and a ruthenium catalyst has been published by Du and Yoon ${ }^{48}$. Irradiation of methyl cinnamate under the conditions as described by these authors, however, failed to give any dimer even after prolonged periods of irradiation (1 week).

Investigation of the reaction mixture by ESI-MS showed (besides products of deterioration) only the presence of unchanged starting material but no dimer. It can be assumed that the substitution pattern of the aromatic system has a high impact on this reaction, since ethyl sinapate (10) afforded in the presence of
$\mathrm{Ru}(\mathrm{bpy})_{3} \mathrm{Cl}_{2}$ hexahydrate dimer $\mathbf{1 1}$, a $\delta$ truxinic acid derivative whose relative configuration was determined by comparison of its ${ }^{1} \mathrm{H}$ NMR spectra with those of compounds of known configuration. Thus, in the ${ }^{1} \mathrm{H}$ NMR spectrum of $\mathbf{1 1}$ an AA'BB' spin system was observed for the cyclobutane protons (being typical for $\delta$-truxinic acid derivatives).

The coupling constants were determined by simulation (see exp. part). Furthermore, for this product of a head-to-head dimerization, in the ESIMS spectra fragments of the quasi-molecular ion $[\mathrm{M}+\mathrm{H}]^{+}$at $\mathrm{m} / \mathrm{z}=253\left(\mathrm{C}_{13} \mathrm{H}_{17} \mathrm{O}_{5}{ }^{+}\right)$and $\mathrm{m} / \mathrm{z}=173$ $\left(\mathrm{C}_{8} \mathrm{H}_{13} \mathrm{O}_{4}{ }^{+}\right)$were observed. Irradiation $(\lambda=470 \mathrm{~nm})$ of sinapin thiocyanate (1) using $\mathrm{Ru}(\mathrm{dndp}){ }_{3} \mathrm{Cl}_{2}$ (dndp $=4,4^{\prime}$-dinonyl-2,2'-dipyridine) ${ }^{49}$ as a catalyst for 5 days gave dimer 12. The latter catalyst proved superior to $\mathrm{Ru}(\mathrm{bpy})_{3} \mathrm{Cl}_{2}$ due to its high solubility in organic solvents. Furthermore, after completion of the reaction, the catalyst can be easily removed by extraction with organic solvents.

The fact that methyl cinnamate does not react under these reaction conditions while the starting materials $\mathbf{1}$ and $\mathbf{1 0}$ lead to the formation of dimeric products $\mathbf{1 1}$ and $\mathbf{1 2}$ is astonishing at first sight. According to the mechanism, however, postulated by Ischay et al. ${ }^{50}$ the triplet state of $\left[\mathrm{Ru}(\mathrm{bpy})_{3}\right]^{2+}$ is decisive for the reaction. If the triplet energy of the molecule is significantly higher than the energy of the catalyst, the reaction will not take place. To get a deeper insight, triplet energies $\left(\mathrm{E}_{\mathrm{T}}(\mathrm{n})\right.$ in $\left.\mathrm{kJ} / \mathrm{mol}\right)$ were calculated (GAUSSIAN) and compared to the triplet energy reported for the ruthenium catalyst. For cinnamic acid as well as for methyl cinnamate triplet energies of $253 \mathrm{~kJ} / \mathrm{mol}$ were found, and for ethyl sinapate (10) and for sinapine (1) energies of ca. $230 \mathrm{~kJ} / \mathrm{mol}$ were calculated.

Furthermore, even lower triplet energy $(166 \mathrm{~kJ} / \mathrm{mol})$ was obtained for the corresponding phenolate of $\mathbf{1 0}$ which may be formed in equilibrium due to the presence of a base (DIPEA) in the reaction medium. For $[\mathrm{Ru}(\mathrm{bpy}) 3]^{2+}$ triplet energy of $208 \mathrm{~kJ} / \mathrm{mol}$ has been reported. From these results, it seems to be plausible why the Ru-catalyst enables dimerization of the sinapic acid derivatives while this is not the case for cinnamic acid and methyl cinnamate.

Biological testing of the dimers [SRB assay for cytotoxicity employing cell lines $\mathrm{FaDu}$ (hypopharyngeal carcinoma), A2780 (ovarian carcinoma), HT29 (colorectal carcinoma), MCF-7 (breast adenocarcinoma), SW1736 (thyroid carcinoma), A375 (melanoma), A549 (epithelial carcinoma) and non-malignant fibroblasts (NIH 3T3); Ellman's assay for cholinesterase inhibition using acetylcholinesterase (from electric eel) or butyrylcholinesterase (from equine serum)] did not reveal any biological activity associated with these dimers. 
<smiles>COc1ccc([C@@H]2[C@H](c3cc(OC)c(OC)c(OC)c3)[C@@H](C(=O)N3CCC=CC3=O)[C@@H]2C(=O)N2CCC=CC2=O)cc1OC</smiles>

Piperarborenine B<smiles>COc1cc(/C=C/C(=O)N2CCC=CC2=O)cc(OC)c1OC</smiles>

Piperlongumine (Piplartine)<smiles>COc1cc([C@@H]2[C@@H](C(=O)N3CCC=CC3=O)[C@H](C(=O)N3CCC=CC3=O)[C@@H]2c2cc(OC)c(OC)c(OC)c2)cc(OC)c1OC</smiles>

Piplartine dimer

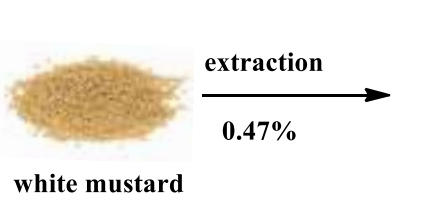<smiles>COc1cc(/C=C/C(=O)OCC[N+](C)([O])[O-])cc(OC)c1O</smiles><smiles>[X]CCN(C)CCCCC[SiH3]</smiles>

i $\left\{\begin{array}{l}6 \mathrm{X}=\mathrm{OH} \\ 7 \mathrm{X}=\mathrm{Cl}\end{array}\right.$<smiles>CCCCCCCCC</smiles><smiles>[R20]c1c(OC)cc(C=O)cc1OC</smiles><smiles>[R]OC(=O)/C=C/c1cc(OC)c(O[GaH2])c(OC)c1</smiles>

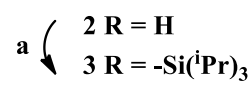<smiles>CCCCCCCCCCC</smiles>

$\mathbf{n}\left\{\begin{array}{l}\mathbf{8} \mathbf{R}=\mathbf{M e} \\ \mathbf{9} \mathbf{R}=\mathbf{H}\end{array}\right.$<smiles>COc1cc(/C=C\C(C)=O)cc(OC)c1O[GaH2]</smiles><smiles>[R]OC(=O)C=Cc1cc(OC)c(O)c(OC)c1</smiles>

$10 \mathrm{R}=\mathrm{OEt}$

$1 \mathrm{R}=\mathrm{O}-\mathrm{CH}_{2}-\mathrm{CH}_{2}-\mathrm{N}\left(\mathrm{CH}_{3}\right)_{3}{ }^{+} \mathrm{SCN}^{-}$<smiles>[R]C(=O)[C@H]1[C@@H](c2cc(OC)c(O)c(OC)c2)[C@H](c2cc(OC)c(O)c(OC)c2)[C@H]1C([R])=O</smiles>

$11 \mathrm{R}=\mathrm{OEt}$

$12 \mathrm{R}=\mathrm{OCH}_{2}-\mathrm{CH}_{2}-\mathrm{N}\left(\mathrm{CH}_{3}\right)_{3}{ }^{+} 2 \mathrm{BF}_{4}^{-}$

Scheme 1. Structure of some naturally occurring cyclobutanes, viz. piperarborenine B, cytotoxic piperlongumine (= piplartine) and an even more cytotoxic synthetic piplartine dimer. Reactions and conditions: a) TEA, DMAP, DMF, DCM, TRIS-Cl, $25^{\circ} \mathrm{C}, 30 \mathrm{~min}, 94 \%$; b) $\mathrm{BrCH}_{2} \mathrm{CO}_{2} \mathrm{Me}, \mathrm{Ph}_{3} \mathrm{P}, \mathrm{NaOH}, \mathrm{DCM}, 25^{\circ} \mathrm{C}, 3 \mathrm{~h}, 88 \%$; c) EtOH, $\mathrm{NaOH}, 60^{\circ} \mathrm{C}, 15 \mathrm{~min}, 84 \%$; d) silica-TLC-plate, hv, $\lambda=254 \mathrm{~nm}, 2 \mathrm{~h}, 25 \%$; e) $\mathrm{K}_{2} \mathrm{CO}_{3}$, dioxane/water, $25^{\circ} \mathrm{C}, 3 \mathrm{~h}$, then 7, DMSO, $70^{\circ} \mathrm{C}, 1$ day, $46 \%$; f) from 10: $\mathrm{Ru}(\mathrm{bpy})_{3} . \mathrm{Cl}_{2}$ hexahydrate, $\mathrm{LiBF}_{4}, \mathrm{MeCN}$, DIPEA, hv, $\lambda=470$ $\mathrm{nm}, 25^{\circ} \mathrm{C}, 8$ days, 65\%; g) from 1, $\mathrm{Ru}(\mathrm{dndp})_{3} \mathrm{Cl}_{2}$, DIPEA, $\mathrm{LiBF}_{4}, \mathrm{~h} v, \lambda=470 \mathrm{~nm}, 25^{\circ} \mathrm{C}, 31 \%$; h) $\mathrm{MeOH}, \mathrm{NaOH}$, $60^{\circ} \mathrm{C}, 15 \mathrm{~min}, 84 \%$; i) $120^{\circ} \mathrm{C} 2 \mathrm{~h}$, then $\mathrm{SOCl}_{2}$, reflux, $1 \mathrm{~h}, 77 \%$.

\section{Conclusion}

A straightforward synthesis for sinapine (1) has been developed starting from commercially available starting materials in an overall yield of $32 \%$ while the extraction of 1 from white mustard gave 1 in $0.47 \%$. Irradiation of $\mathbf{1}$ in the presence of $\mathrm{Ru}(\mathrm{dndp}){ }_{3} \mathrm{Cl}_{2}$ furnished in a [2+2] cycloaddition cyclobutanoid dimer 12, a $\delta$-truxinic acid. Thereby, this catalyst proved superior to $\mathrm{Ru}(\mathrm{bpy})_{3} \mathrm{Cl}_{2}$ due it high solubility 
in organic solvents. The putative mechanism of this reaction was supported by DFT calculations. In contrast to piplartine dimers, the dimer derived from sinapine did not show any cytotoxic activity for a variety of human tumor cell lines.

\section{Acknowledgments}

Many thanks are due to Dr. D. Ströhl and his team for the NMR spectra. IR- and UV-vis spectra were recorded by Ms V. Simon. Biological assays were performed by $A$. Loesche and S. Hoenke; experimental help in the lab was provided by A.M. Deckelmann, J. Wiese and T.L.T. Luong.

\section{Experimental}

\subsection{General}

The reagents were bought from commercial suppliers and used without further purification. Mustard seeds (Waldenheimer Gewürze) were purchased from a local supermarket. The solvents were dried according to usual procedures. For the photolysis, the diode LIU470A (470 nm, 1 W) from Thorlabs was used. Melting points were determined on Büchi Melting Point M-565 or LEICA hot stage microscope and are uncorrected, NMR spectra were recorded on Agilent $400 \mathrm{MHz}$ VNMRS and $500 \mathrm{MHz}$ DD2 spectrometers ( $\delta$ given in ppm, $J$ in $\mathrm{Hz}$ ), ESI mass spectra were obtained on a Finnigan MAT LCQ (spray voltage 4.1 $\mathrm{kV}$, sheath gas nitrogen) instrument. Macherey-Nagel ALUGRAM $^{\circledR}$ Xtra SIL G/UV 254 pre-coated silica gel 60 F254 plates were used for thin-layer chromatography (detection with cerium molybdate spray reagent and UV absorption). IR spectra were recorded on a Perkin-Elmer FT-IR spectrometer Spectrum 1000 and wavenumbers are expressed in $\mathrm{cm}^{-1}$. The absorption spectra were measured on Perkin Elmer Lambda14 spectrometer. Microanalyses were performed with an Elementar Vario EL (CHNS) instrument.

\subsection{Quantum chemical calculations}

The triplet energies were calculated as vertical excitation energies by time-dependent DFT with the Gaussian 09 package using the B3LYP functional 6-311++G(2d,2p) basis set, and as solvation model IEFPCM with the solvent acetonitrile after optimization of the ground state geometry.

Sinapine thiocyanate: $(\boldsymbol{E}) \mathrm{2}-[(\mathbf{3}-(\mathbf{4}-\mathbf{h y d r o x y}-\mathbf{3 , 5}-$
dimethoxyphenyl)acryloyl)oxy]- $\boldsymbol{N}, \boldsymbol{N}, \boldsymbol{N}$ -
trimethylethan-1-aminium thiocyanate (1)
From 5: Compound $\mathbf{5}(0.19 \mathrm{~g}, 0.49 \mathrm{mmol})$ was
dissolved in a dioxane-water-mixture $(3: 2,5 \mathrm{~mL})$, and
a solution of $\mathrm{K}_{2} \mathrm{CO}_{3}(0.03 \mathrm{~g}, 0.24 \mathrm{mmol})$ in water
$(1 \mathrm{~mL})$ was added, and stirring at room temperature
was continued for another $3 \mathrm{~h}$. The volatiles were
removed under reduced pressure. Chlorocholine
chloride $(7,0.07 \mathrm{~g}, 0.56 \mathrm{mmol})$ was added, and the
solids were dried at $70^{\circ} \mathrm{C}$ under reduced pressure for
$1 \mathrm{~h}$. Dry DMSO $(5 \mathrm{~mL})$ was added, and the mixture was stirred at $60^{\circ} \mathrm{C}$ for 2 days. The solvent was removed under reduced pressure, and the residue was washed with chloroform ( $3 \times 4 \mathrm{~mL})$, dissolved in water $(4 \mathrm{~mL})$ and extracted with chloroform $(4 \mathrm{~mL})$. To the aqueous phase, an aqueous solution of KSCN $(20 \%, 25 \mathrm{~mL})$ was added, and the mixture was allowed to stand at $0^{\circ} \mathrm{C}$ for $48 \mathrm{~h}$. The precipitate was filtrated off and recrystallized from ethanol. Compound $1(0.08 \mathrm{~g}, 46 \%)$ was obtained as a colorless solid; m.p. $178-180^{\circ} \mathrm{C}$; m.m.p. $178-180^{\circ} \mathrm{C}$.

By extraction: Mustard seeds (330 g) were crushed with a kitchen mill and extracted in a Soxhlet apparatus with $n$-hexane $(900 \mathrm{~mL})$ for $8 \mathrm{~h}$. The dried grist was extracted with $70 \%$ methanol for $1 \mathrm{~h}$ at $60^{\circ} \mathrm{C}$ (ultrasound-assisted) and filtered. The methanol was removed under reduced pressure, and the aqueous phase was washed with chloroform (4 x $100 \mathrm{~mL})$. To the resulting aqueous phase an aqueous solution of $\operatorname{KSCN}(20 \%, 200 \mathrm{~mL})$ was added, and the mixture was allowed to rest at $0^{\circ} \mathrm{C}$ for 2 days. The precipitate was filtrated off and recrystallized from ethanol to yield $1(1.56 \mathrm{~g}, 0.47 \%)$ as a colorless solid; $\mathrm{R}_{\mathrm{F}}=0.35$ (silica gel, $n$-butanol/ $n$-propanol/water 1:1:1); m.p. $178-180^{\circ} \mathrm{C}$ (lit.: $178^{\circ} \mathrm{C}^{51}$ );

IR $(\mathrm{KBr}):=3448 w, 2060 \mathrm{~s}, 1710 \mathrm{~m}, 1637 \mathrm{~s}, 1516 \mathrm{~m}$, $1457 \mathrm{~m}, 1336 \mathrm{w}, 1168 \mathrm{~s}, 1110 \mathrm{~m} \mathrm{~cm}^{-1}$;

UV-vis $\left(\mathrm{H}_{2}\right) \mathrm{O}: \lambda(\log \varepsilon)=243(3.82), 355(3.88) \mathrm{nm}$; ${ }^{1} \mathrm{H}$ NMR (400 MHz, DMSO-d 6 ): $\delta=9.03(s, 1 \mathrm{H}, \mathrm{OH})$, $7.60(d, J=15.9 \mathrm{~Hz}, 1 \mathrm{H}, 3-\mathrm{H}), 7.03(s, 2 \mathrm{H}$, arom.), $6.55(d, J=15.9 \mathrm{~Hz}, 1 \mathrm{H}, 2-\mathrm{H}), 4.57\left(m, 2 \mathrm{H}, \mathrm{OCH}_{2}\right)$, $3.80\left(s, 6 \mathrm{H}, 2 \times \mathrm{OCH}_{3}\right), 3.74-3.69\left(m, 2 \mathrm{H}, \mathrm{NCH}_{2}\right)$, $3.17\left(s, 9 \mathrm{H}, \mathrm{NMe}_{3}^{+}\right) \mathrm{ppm}$;

${ }^{13} \mathrm{C}$ NMR (100 MHz, DMSO-d 6$): \delta=165.9(\mathrm{C}=\mathrm{O})$, 148.1 (C, $2 \mathrm{x}$ arom. $\left.C-\mathrm{OCH}_{3}\right), 146.2$ (C-3), 138.7 (C, arom. $C-\mathrm{OH}), 129.5\left(\mathrm{SCN}^{-}\right), 124.2(\mathrm{C}$, arom. $), 114.1$ (C-2), 106.5 (2 x CH, arom.), $64.0\left(\mathrm{OCH}_{2}\right), 57.7$ $\left(\mathrm{NCH}_{2}\right), 56.2\left(2 \times \mathrm{OCH}_{3}\right), 53.0\left(\mathrm{NMe}_{3}{ }^{+}\right) \mathrm{ppm}$; MS (ESI, MeOH): m/z $(\%)=251.1([\mathrm{M}-\mathrm{SCN}$, $\left.\left.-\mathrm{NMe}_{3}\right]^{+}, 24\right), 310.1\left([\mathrm{M}-\mathrm{SCN}]^{+}, 100\right)$; analysis calcd for $\mathrm{C}_{17} \mathrm{H}_{24} \mathrm{~N}_{2} \mathrm{O}_{5} \mathrm{~S}$ (368.45): C 55.42, H 6.57, N 7.60, S 8.70; found: C 55.18, H 6.79, N 7.36, S 8.56.

\section{3,5-Dimethoxy-4-[(triisopropylsilyl)oxy]- benzaldehyde (3)}

To a solution of 3,5-dimethoxy-4hydroxybenzaldehyde $(2,0.60 \mathrm{~g}, 3.3 \mathrm{mmol})$, triethylamine $(0.6 \mathrm{~mL}, 4.0 \mathrm{mmol})$ and DMAP (catalytic) in dry DMF $(5 \mathrm{~mL})$, a solution of triisopropylsilylchloride $(0.9 \mathrm{~mL}, 4.0 \mathrm{mmol})$ in dry DCM ( $2 \mathrm{~mL})$ was added, and the mixture was stirred at $25^{\circ} \mathrm{C}$ for $30 \mathrm{~min}$ at room temperature. Usual aqueous workup followed by chromatography (flash, silica gel, $n$-hexane/ethyl acetate 9:1) gave 3 (1.05 g, $94 \%$ ) as a slightly yellowish liquid; $\mathrm{R}_{\mathrm{F}}=0.54$ (silica gel, $n$-hexane/ethyl acetate 9:1);

IR $($ KBR) $v=3449 w, 2944 m, 2867 m, 2361 w, 1693 m$, $1584 m, 1507 s, 1464 m, 1424 w, 1389 w, 1337 s, 1268 w$, $1231 w, 1131 \mathrm{~s} \mathrm{~cm}^{-1}$;

$\mathrm{UV}$-vis $\left(\mathrm{CHCl}_{3}\right): \lambda(\log \varepsilon)=232$ (4.09),

$311(4.09) \mathrm{nm}$; 
${ }^{1} \mathrm{H}$ NMR $\left(500 \mathrm{MHz}, \mathrm{CDCl}_{3}\right): \delta=9.81(s, 1 \mathrm{H}, 1-\mathrm{H}$, $\mathrm{CHO}), 7.08\left(s, 2 \mathrm{H}\right.$, arom.), $3.85\left(s, 6 \mathrm{H}, 2 \times \mathrm{OCH}_{3}\right)$, $1.25(m, 3 \mathrm{H}, 3 \times \mathrm{CH}-\mathrm{Si}), 1.07(d, J=7.4 \mathrm{~Hz}, 18 \mathrm{H}, 6 \mathrm{x}$ $\left.\mathrm{CH}_{3}\right) \mathrm{ppm}$;

${ }^{13} \mathrm{C} \mathrm{NMR}\left(125 \mathrm{MHz}, \mathrm{CDCl}_{3}\right): \delta=191.5(\mathrm{CH}, \mathrm{C}=\mathrm{O})$, $151.8\left(\mathrm{C}, 2 \mathrm{x}\right.$ arom. $\left.C-\mathrm{OCH}_{3}\right), 141.3(\mathrm{C}$, arom. $C$-OSi $), 128.8$ (C, arom.), 106.6 (2 x CH, arom.), 55.6 $\left(2 \times \mathrm{OCH}_{3}\right), 17.8\left(6 \times \mathrm{CH}_{3}\right), 13.9(\mathrm{CH}, 3 \times \mathrm{CH}-\mathrm{Si})$ ppm;

MS (ESI, $\mathrm{MeOH}): \mathrm{m} / \mathrm{z}(\%)=339.1\left([\mathrm{M}+\mathrm{H}]^{+}, 100\right)$; analysis calcd for $\mathrm{C}_{18} \mathrm{H}_{30} \mathrm{O}_{4} \mathrm{Si}$ (338.52): C 63.87, H 8.93; found: C 63.55, H 9.15.

\section{Methyl (E) 3-(3,5-dimethoxy-4- \\ ((triisopropylsilyl)oxy)phenyl) acrylate (4)}

To a solution of $3(0.2 \mathrm{~g}, 0.6 \mathrm{mmol})$ in dry DCM $(4 \mathrm{~mL})$, methyl bromoacetate $(0.08 \mathrm{~mL})$ and $\mathrm{PPh}_{3}(0.2$ g, $0.9 \mathrm{mmol})$ were added. An aqueous solution of $\mathrm{NaOH}(7 \mathrm{~mL}, 0.5 \mathrm{M})$ was slowly added, and the reaction mixture was stirred for $3 \mathrm{~h}$ at room temperature. Usual aqueous work-up followed by column chromatography (silica gel, $n$-hexane/ethyl acetate, 9:1) gave $4(0.18 \mathrm{~g}, 88 \%)$ as a colorless solid; $\mathrm{R}_{\mathrm{F}}=0.43$ (silica gel, $n$-hexane/ethyl acetate, 9:1); m.p. $84-87^{\circ} \mathrm{C}$;

IR $(\mathrm{KBr}): \mathrm{v}=3447 w, 2943 m, 2865 m, 1699 s, 1631 w$, $1579 m, 1508 s, 1464 m, 1424 w, 1344 m, 1266 m$, $1248 w, 1161 w, 1130 \mathrm{~s} \mathrm{~cm}^{-1}$;

UV-vis $\left(\mathrm{CHCl}_{3}\right): \lambda(\log \varepsilon)=236$ (4.27), 324 (4.32) nm;

${ }^{1} \mathrm{H}$ NMR $\left(500 \mathrm{MHz}, \mathrm{CDCl}_{3}\right): \delta=7.60(d, J=15.9 \mathrm{~Hz}$, $1 \mathrm{H}, 3-\mathrm{H}), 6.71(s, 2 \mathrm{H}$, arom.), $6.29(d, J=15.9 \mathrm{~Hz}$, $1 \mathrm{H}, 2-\mathrm{H}), 3.81\left(s, 6 \mathrm{H}, 2 \times \mathrm{OCH}_{3}\right), 3.79\left(s, 3 \mathrm{H}, \mathrm{OCH}_{3}\right)$, $1.24(m, 3 \mathrm{H}, 3 \times \mathrm{CH}-\mathrm{Si}), 1.10(d, J=7.4 \mathrm{~Hz}, 18 \mathrm{H}, 3 \mathrm{x}$ $\left.\mathrm{CH}_{3}\right) \mathrm{ppm} ;{ }^{13} \mathrm{C} \mathrm{NMR}\left(125 \mathrm{MHz}, \mathrm{CDCl}_{3}\right): \delta=167.7$ $(\mathrm{C}-1, \mathrm{C}=\mathrm{O}), 151.8\left(\mathrm{C}, 2 \mathrm{x}\right.$ arom. $\left.C-\mathrm{OCH}_{3}\right), 145.3(\mathrm{CH}$, C-3), 137.9 (C, arom. $C$-OSi), 127.1 (C, arom.), 115.7 $(\mathrm{CH}, \mathrm{C}-2), 105.5\left(\mathrm{CH}\right.$, arom.), $55.6\left(2 \times \mathrm{OCH}_{3}\right), 51.6$ $\left(\mathrm{OCH}_{3}\right), 17.8\left(3 \times \mathrm{CH}_{3}\right), 13.3(3 \times \mathrm{CH}-\mathrm{Si}) \mathrm{ppm}$; MS (ESI, MeOH): m/z (\%) = $395.1\left([\mathrm{M}+\mathrm{H}]^{+}, 100\right)$; analysis calcd for $\mathrm{C}_{21} \mathrm{H}_{34} \mathrm{O}_{5} \mathrm{Si}$ (394.58): C 63.92, H 8.69; found: C 63.77, H 8.92.

\section{(E) 3-(3,5-Dimethoxy-4-((triisopropylsilyl)oxy) phenyl)-acrylic acid (5)}

To a solution of $4(1.5 \mathrm{~g}, 4.3 \mathrm{mmol})$ in ethanol $(20 \mathrm{~mL})$ and $\mathrm{NaOH}(2 \mathrm{M}, 8.5 \mathrm{~mL})$ was stirred for 15 $\min$ at $60^{\circ} \mathrm{C}$. The precipitate was dissolved in water ( $3 \mathrm{~mL})$, and diluted $\mathrm{HCl}(2 \mathrm{M}, 6 \mathrm{~mL})$ was added. The precipitate was filtered off and dried; compound $\mathbf{5}$ $(1.36 \mathrm{~g}, 84 \%)$ was obtained as a white solid; $\mathrm{R}_{\mathrm{F}}=0.55$ (silica gel, $n$-hexane/ethyl acetate, 9:1); m.p. $149-152^{\circ} \mathrm{C}$;

IR $(\mathrm{KBr}): \mathrm{v}=3447 s s, 2944 w, 2361 w, 1685 w, 1631 \mathrm{~m}$, $1582 w, 1509 s, 1458 w, 1288 m, 1160 w, 1133 \mathrm{~m} \mathrm{~cm}^{-1}$; UV-vis $\left(\mathrm{CHCl}_{3}\right): \lambda(\log \varepsilon)=237$ (4.27), 321 (4.16) $\mathrm{nm}$;

${ }^{1} \mathrm{H}$ NMR (500 MHz, $\left.\mathrm{CD}_{3} \mathrm{OD}\right): \delta=7.61(d, J=15.9$ $\mathrm{Hz}, 1 \mathrm{H}, 3-\mathrm{H}), 6.88$ ( $s, 2 \mathrm{H}$, arom.), 6.38 ( $d, J=15.9$ $\mathrm{Hz}, 1 \mathrm{H}, 2-\mathrm{H}), 3.83\left(s, 6 \mathrm{H}, 2 \times \mathrm{OCH}_{3}\right), 1.26(m, 3 \mathrm{H}$, 3 x CH-Si), 1.09 ( $\left.d, J=7.4 \mathrm{~Hz}, 18 \mathrm{H}, 3 \times \mathrm{CH}_{3}\right) \mathrm{ppm}$;
${ }^{13} \mathrm{C}$ NMR (125 MHz, $\left.\mathrm{CD}_{3} \mathrm{OD}\right): \delta=170.8(\mathrm{C}-1, \mathrm{C}=\mathrm{O})$, $153.1\left(\mathrm{C}, 2 \mathrm{x}\right.$ arom. $\left.C-\mathrm{OCH}_{3}\right), 147.1(\mathrm{CH}, \mathrm{C}-3), 138.5$ (C, arom. $C$-OSi), 128.6 (C, arom.), 117.1 (CH, C-2), 106.5 (CH, arom.), $56.2\left(2 \times \mathrm{OCH}_{3}\right), 18.7\left(6 \times \mathrm{CH}_{3}\right)$, 15.0 (3 x CH-Si) ppm;

MS (ESI, $\mathrm{MeOH}): \mathrm{m} / \mathrm{z}(\%)=381.1\left([\mathrm{M}+\mathrm{H}]^{+}, 100\right)$; analysis calcd for $\mathrm{C}_{20} \mathrm{H}_{32} \mathrm{O}_{5} \mathrm{Si}$ (380.55): C 63.12, H 8.48; found: 62.95, H 8.69.

\section{2-Chloro- $N, N, N$-trimethyl-ethanaminium chloride (7)}

Commercial cholin chloride $(6,2.89 \mathrm{~g}, 20.7 \mathrm{mmol})$ was dried in vacuo at $120^{\circ} \mathrm{C}$ for $2 \mathrm{~h}$. Freshly distilled thionyl chloride $(20 \mathrm{~mL})$ was added, and the mixture was heated under reflux for $1 \mathrm{~h}$. The volatiles were removed under diminished pressure, methanol (20 $\mathrm{mL} ; 3 \mathrm{x}$ ) was added, and removed under diminished pressure. The remaining solid was dried at $80^{\circ} \mathrm{C}$ under reduced pressure to afford $7(2.45 \mathrm{~g}, 77 \%)$ as a colorless solid; $\mathrm{R}_{\mathrm{F}}=0.44$ (RP18-silica gel, $\mathrm{MeCN} /$ water, $10: 1$ ); m.p. $240^{\circ} \mathrm{C}$ (decomp.; lit.: $\left.240^{\circ} \mathrm{C}^{52}\right)$

IR (KBR): $v=3449 s, 3006 m, 2362 w, 1636 w$, $1482 \mathrm{~s} \mathrm{~cm}^{-1}$;

${ }^{1} \mathrm{H}$ NMR (500 MHz, CD $\left.{ }_{3} \mathrm{OD}\right): \delta=3.24(s, 9 \mathrm{H}, 3 \mathrm{x}$ $\left.\mathrm{CH}_{3}\right), 3.81\left(t, J=7.2 \mathrm{~Hz}, 2 \mathrm{H}, \mathrm{N}-\mathrm{CH}_{2}\right), 4.06(t, J=7.2$ $\left.\mathrm{Hz}, 2 \mathrm{H}, \mathrm{Cl}-\mathrm{CH}_{2}\right) \mathrm{ppm}$;

${ }^{13} \mathrm{C}$ NMR (125 MHz, CD $\left.{ }_{3} \mathrm{OD}\right): \delta=67.7\left(\mathrm{~N}^{-\mathrm{CH}_{2}}\right)$, $54.3\left(\mathrm{~N}-\mathrm{CH}_{3}\right), 36.7\left(\mathrm{Cl}-\mathrm{CH}_{2}\right) \mathrm{ppm}$;

MS (ESI, $\mathrm{MeOH}): \mathrm{m} / \mathrm{z}(\%)=122.1\left([\mathrm{M}-\mathrm{Cl}]^{+}, 100\right)$.

Methyl (Z) 3-(3,5-dimethoxy-4-[(triisopropylsilyl)oxy]-phenyl) acrylate (8)

A solution of $4(0.2 \mathrm{~g}, 0.5 \mathrm{mmol})$ in chloroform $(2 \mathrm{~mL})$ was applied onto a silica gel TLC plate and irradiated for $2 \mathrm{~h}(\lambda=2154 \mathrm{~nm})$. The silica gel was removed from the plate, extracted with methanol, filtered, and the filtrate was evaporated. The residue was subjected to chromatography (silica gel, $n$-hexane/ethyl acetate, $9: 1)$ to yield $\mathbf{8}(0.05 \mathrm{~g}, 25 \%)$ as a viscous oil; $\mathrm{R}_{\mathrm{F}}=0.68$ (silica gel, $n$-hexane/ethyl acetate, 9:1);

IR (film): $v=3442 \mathrm{br}, 2946 \mathrm{~m}, 2866 \mathrm{~m}, 1720 \mathrm{~m}, 1624 \mathrm{~m}$, $1578 m, 1512 s, 1464 m, 1420 w, 1344 m, 1246 m, 1132 s$,

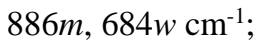

UV-vis $(\mathrm{CHCl} 3): \lambda_{\max }(\log \varepsilon)=232$ (4.27), 321 (3.95) $\mathrm{nm}$;

${ }^{1} \mathrm{H}$ NMR (500 MHz, $\left.\mathrm{CDCl}_{3}\right): \delta=7.13$ (s, 2H, arom.), $6.78(d, J=12.9 \mathrm{~Hz}, 1 \mathrm{H}, \mathrm{CH}=), 5.82(d, J=12.9 \mathrm{~Hz}$, $\mathrm{CH}=), 3.82\left(s, 6 \mathrm{H}, 2 \times \mathrm{OCH}_{3}\right), 3.73\left(s, 3 \mathrm{H}, \mathrm{CO}_{2} \mathrm{CH}_{3}\right)$, 1.26 (sept., $J=7.3 \mathrm{~Hz}, 3 \mathrm{H}, 3 \times \mathrm{CH}-\mathrm{Si}), 1.09(d, J=$ $\left.7.3 \mathrm{~Hz}, 18 \mathrm{H}, 6 \times \mathrm{CH}_{3}\right) \mathrm{ppm}$;

${ }^{13} \mathrm{C} \mathrm{NMR}\left(125 \mathrm{MHz}, \mathrm{CDCl}_{3}\right): \delta=166.8(\mathrm{C}-1, \mathrm{C}=\mathrm{O})$, $150.6\left(\mathrm{C}, 2 \mathrm{x}\right.$ arom. $\left.C-\mathrm{OCH}_{3}\right), 143.8(\mathrm{CH}, \mathrm{C}-3), 136.7$ (C, arom. C-OSi), $116.3(\mathrm{CH}, \mathrm{C}-2), 108.1(\mathrm{CH}$, arom.), $55.6\left(2 \mathrm{x} \quad \mathrm{OCH}_{3}\right), 51.3\left(\mathrm{CO}_{2} \mathrm{CH}_{3}\right), 17.9$ (6 x $\left.\mathrm{CH}_{3}\right), 13.3$ (3 x CH-Si) ppm;

MS (ESI, MeOH): m/z (\%) = $395.1\left([\mathrm{M}+\mathrm{H}]^{+}, 100\right)$; analysis calcd. for $\mathrm{C}_{21} \mathrm{H}_{34} \mathrm{O}_{5} \mathrm{Si}$ (394.58): C 63.92, H 8.69; found: C 63.65, H 8.96. 


\section{(Z) 3-(3,5-Dimethoxy-4-[(triisopropylsilyl)oxy] phenyl)-acrylic acid (9)}

To a solution of $8(0.18 \mathrm{~g}, 0.47 \mathrm{mmol})$ in methanol $(5 \mathrm{~mL})$, an aqueous solution of $\mathrm{NaOH}(2 \mathrm{M}, 1.0 \mathrm{ml})$ was added, and stirring at $60^{\circ} \mathrm{C}$ was continued for 15 min. The precipitate was suspended in water $(3 \mathrm{~mL})$ and aq. hydrochloric acid ( $2 \mathrm{M}, 4 \mathrm{~mL})$ was added. The precipitate was filtered off and re-crystallized from 2-propanol to yield $9(0.15 \mathrm{~g}, 84 \%)$ as a colorless solid; m.p. $86-89^{\circ} \mathrm{C} ; \quad \mathrm{R}_{\mathrm{F}}=0.61$ (silica gel, $\left.\mathrm{CHCl}_{3} / \mathrm{MeOH}, 9: 1\right)$;

IR (KBr): $v=3452 s, 2941 m, 2886 m, 1684 w, 1613 m$, $1575 m, 1512 s, 1464 s, 1422 m, 1348 m, 1236 m, 1130 s$ $\mathrm{cm}^{-1}$; UV-vis $(\mathrm{MeOH}): 1_{\max }(\log \varepsilon)=204$ (4.31), 313 (3.92) $\mathrm{nm}$;

${ }^{1} \mathrm{H} \mathrm{NMR}\left(500 \mathrm{MHz}, \mathrm{CD}_{3} \mathrm{OD}\right): \delta=7.16(s, 2 \mathrm{H}$, arom. $)$, $6.83(d, J=12.9 \mathrm{~Hz}, 1 \mathrm{H}, \mathrm{CH}=), 5.85(d, J=12.9 \mathrm{~Hz}$, $1 \mathrm{H}, \mathrm{CH}=), 3.80\left(\mathrm{~s}, 6 \mathrm{H}, 2 \times \mathrm{OCH}_{3}\right), 1.27$ (sept., $J=7.2$ $\mathrm{Hz}, 3 \mathrm{H}, 3 \times \mathrm{CH}-\mathrm{Si}), 1.10\left(d, J=7.2 \mathrm{~Hz}, 6 \times \mathrm{CH}_{3}\right) \mathrm{ppm}$; ${ }^{13} \mathrm{C} \mathrm{NMR}\left(125 \mathrm{MHz}, \mathrm{CD}_{3} \mathrm{OD}\right): \delta=169.5(\mathrm{C}-1, \mathrm{C}=\mathrm{O})$, $151.6\left(\mathrm{C}, 2\right.$ x arom. $\left.C-\mathrm{OCH}_{3}\right), 143.7(\mathrm{CH}, \mathrm{C}-3), 136.9$ (C, arom. C-OSi), 128.1 (arom.), $118.2(\mathrm{CH}, \mathrm{C}-2)$, $108.5\left(\mathrm{CH}\right.$, arom.), $55.5\left(2 \times \mathrm{OCH}_{3}\right), 17.7\left(6 \times \mathrm{CH}_{3}\right)$, 13.8 ( 2 x CH-Si) ppm;

MS (ESI, MeOH): m/z $(\%)=381.1\left([\mathrm{M}+\mathrm{H}]^{+}, 100\right)$; analysis calcd for $\mathrm{C}_{20} \mathrm{H}_{32} \mathrm{O}_{5} \mathrm{Si}$ (380.56): C 63.12, $\mathrm{H}$ 8.48; found: 62.90, H 8.72.

Ethyl (E) 3-(4-hydroxy-3,5-dimethoxyphenyl)acrylate $^{[17]}(10)$

Sinapic acid (2.0 g, $8.92 \mathrm{mmol})$ was dissolved in EtOH $(10 \mathrm{~mL})$ and sulfuric acid $(0.09 \mathrm{~mL}, 8.92 \mathrm{mmol})$ was added. The reaction mixture was stirred under reflux for $6 \mathrm{~h}$. The solvent was removed in vacuo, and the residue was suspended in cold water. The aqueous phase was washed with ethyl acetate $(3 \times 50 \mathrm{~mL})$, $\mathrm{NaHCO}_{3}$-solution $(2 \times 20 \mathrm{~mL})$ and brine $(1 \times 50 \mathrm{~mL})$. The organic phase was dried over $\mathrm{MgSO}_{4}$, and the solvent was removed under reduced pressure. Compound $\mathbf{1 0}$ was obtained as an off-white solid (2.0 g, 89\%); $\mathrm{R}_{\mathrm{F}}=0.27$ (silica gel, chloroform); m.p. 80-82 ${ }^{\circ} \mathrm{C}$ (lit.: ${ }^{18} 83^{\circ} \mathrm{C}$ );

IR (KBr): $v=3506 m, 2940 w, 2834 w, 1692 s, 1633 m$, $1609 m, 1517 s, 1468 m, 1429 s, 1368 w, 1336 s, 1289 s$, $1201 s, 1157 \mathrm{~s}, 1106 \mathrm{~s}, 1045 \mathrm{~m}, 978 \mathrm{~m}, 819 \mathrm{~m} \mathrm{~cm}^{-1}$; UV-vis $\left(\mathrm{CHCl}_{3}\right): \lambda(\log \varepsilon)=259(3.81)$,

354 (4.28) nm;

${ }^{1} \mathrm{H} \mathrm{NMR}\left(500 \mathrm{MHz}, \mathrm{CDCl}_{3}\right): \delta=7.59(d, J=15.9 \mathrm{~Hz}$, $1 \mathrm{H}, \mathrm{CH}=), 6.77(s, 2 \mathrm{H}$, arom.), $6.30(d, J=15.9 \mathrm{~Hz}$, $1 \mathrm{H}, \mathrm{CH}=), 4.26\left(q, J=7.1 \mathrm{~Hz}, 2 \mathrm{H}, \mathrm{O}-\mathrm{CH}_{2}\right), 3.92$ $\left(s, 6 \mathrm{H}, 2 \times \mathrm{OCH}_{3}\right), 1.34\left(t, J=7.1 \mathrm{~Hz}, 3 \mathrm{H}, \mathrm{CH}_{3}\right) \mathrm{ppm}$; ${ }^{13} \mathrm{C}$ NMR $\left(125 \mathrm{MHz} ; \mathrm{CDCl}_{3}\right): \delta=167.3(\mathrm{C}-1, \mathrm{C}=\mathrm{O})$, $147.4\left(\mathrm{C}, 2 \mathrm{x}\right.$ arom. $\left.C-\mathrm{OCH}_{3}\right), 145.0(\mathrm{CH}, \mathrm{C}-3), 137.2$ (C, arom. $C-\mathrm{OH}), 126.1$ (C, arom.), $116.2(\mathrm{CH}, \mathrm{C}-2)$, $105.2(\mathrm{CH}$, arom. $), 60.5\left(\mathrm{OCH}_{2}\right), 56.5\left(2 \times \mathrm{OCH}_{3}\right)$, $14.5\left(\mathrm{CH}_{3}\right) \mathrm{ppm}$;

MS (ESI, $\mathrm{MeOH}): \mathrm{m} / \mathrm{z}(\%)=207.1\left([\mathrm{M}+\mathrm{H}-\mathrm{EtOH}]^{+}\right.$, 100), $253.0\left([\mathrm{M}+\mathrm{H}]^{+}, 91\right), 275.1\left([\mathrm{M}+\mathrm{Na}]^{+}, 32\right)$.

Diethyl (1RS,2RS,3SR,4SR)-3,4-Bis(4-hydroxy3,5-dimethoxyphenyl)cyclobutan-1,2dicarboxylate (11)
To a solution of $\mathbf{1 0}(0.41 \mathrm{~g}, 1.62 \mathrm{mmol}), \mathrm{Ru}(\mathrm{bpy})_{3} \mathrm{Cl}_{2}$ - $6 \mathrm{H}_{2} \mathrm{O}(0.017 \mathrm{~g}, 0.023 \mathrm{mmol})$ and $\mathrm{LiBF}_{4}(0.172 \mathrm{~g}$, $1.82 \mathrm{mmol})$ in dry acetonitrile $(5 \mathrm{~mL})$, DIPEA $(0.157$ $\mathrm{mL}, 0.119 \mathrm{~g}, 0.907 \mathrm{mmol})$ was added. The reaction mixture was irradiated $(\lambda=470 \mathrm{~nm})$ for 8 days at room temperature. The solvent was removed under reduced pressure, and the residue was dissolved in chloroform $(80 \mathrm{~mL})$. The organic phase was washed with water (3 x $40 \mathrm{~mL})$, brine $(1 \times 40 \mathrm{~mL})$ and was dried over $\mathrm{MgSO}_{4}$. The raw product was purified by column chromatography (silica gel, toluene/chloroform/acetone, 5:3:2), and compound $\mathbf{1 1}$ was obtained as an off-white solid $(0.266 \mathrm{~g}, 65 \%)$; $\mathrm{R}_{\mathrm{F}}=0.39$ (silica gel, toluene/chloroform/acetone, 5:3:2); m.p. $129^{\circ} \mathrm{C}$;

IR (KBr): $v=3425 w, 2939 w, 1723 m, 1611 m, 1518 m$, $1455 m, 1369 w, 1339 m, 1322 m, 1247 m, 1208 s, 1105 s$, $1018 m, 833 m, 820 m, 734 m, 635 m, 586 m, 534 m \mathrm{~cm}^{-1}$; UV-vis $\left(\mathrm{CHCl}_{3}\right): \lambda(\log \varepsilon)=260$ (4.81), 358 (3.70) $\mathrm{nm}$;

${ }^{1} \mathrm{H}$ NMR $\left(400 \mathrm{MHz}, \mathrm{CDCl}_{3}\right): \delta=6.53(s, 4 \mathrm{H}$, arom. $\mathrm{CH}), 5.44(s, 2 \mathrm{H}, 2 \times \mathrm{OH}), 4.21(q, J=7.1 \mathrm{~Hz}, 4 \mathrm{H}, 2$ $\left.\mathrm{x} \mathrm{OCH}_{2}\right), 3.86\left(s, 12 \mathrm{H}, 4 \mathrm{x} \mathrm{OCH}_{3}\right), 3.57,3.39$ $\left(A A^{\prime} B B^{\prime},{ }^{3} J_{A A^{\prime}}=9.7 \mathrm{~Hz},{ }^{3} J_{B B^{\prime}}=9.6 \mathrm{~Hz},{ }^{3} J_{A B}={ }^{3} J_{A^{\prime} B^{\prime}}=\right.$ $9.3 \mathrm{~Hz},{ }^{4} J_{A B^{\prime}}={ }^{4} J_{A^{`} B}=-0.2 \mathrm{~Hz}, 4 \mathrm{H}$, cyclobutane $\left.\mathrm{H}^{`} \mathrm{~s}\right)$, $1.28\left(t, J=7.1 \mathrm{~Hz}, 6 \mathrm{H}, 2 \times \mathrm{CH}_{3}\right) \mathrm{ppm}$;

${ }^{13} \mathrm{C} \mathrm{NMR}\left(100 \mathrm{MHz} ; \mathrm{CDCl}_{3}\right): \delta=172.8(2 \times \mathrm{C}=\mathrm{O})$, $147.2\left(\mathrm{C}, 4 \mathrm{x}\right.$ arom. $\left.C-\mathrm{OCH}_{3}\right), 134.0(\mathrm{C}, 2 \mathrm{x}$ arom. $C-\mathrm{OH}), 132.6$ ( $2 \times \mathrm{C}$, arom.), 103.6 (4 x CH, arom. ), $61.2\left(2 \times \mathrm{OCH}_{2}\right), 56.5\left(4 \times \mathrm{OCH}_{3}\right), 48.0(2 \times \mathrm{CH}$, C-3, C-4), 44.8 (2 x CH, C-1, C-2), $14.4\left(2 \times \mathrm{CH}_{3}\right)$ ppm;

MS (ESI, MeOH): m/z $(\%)=459.1\left([\mathrm{M}+\mathrm{H}-\mathrm{EtOH}]^{+}\right.$, 38), $504.9\left([\mathrm{M}+\mathrm{H}]^{+}, 100\right), 521.9\left(\left[\mathrm{M}+\mathrm{NH}_{4}\right]^{+}, 23\right)$, $527.1\left([\mathrm{M}+\mathrm{Na}]^{+}, 39\right)$;

analysis calcd for $\mathrm{C}_{26} \mathrm{H}_{32} \mathrm{O}_{10}$ (504.53): C 61.90, H 6.39; found C 61.65, H 6.61.

\section{2,2'-(((1RS,2RS,3SR,4SR)-3,4-Bis(4-hydroxy-3,5- dimethoxyphenyl)-cyclobutan-1,2-dicarbonyl)- $\operatorname{bis}(\operatorname{oxy}))$-bis( $N, N, N$-trimethylethan-1-aminium) bis(tetrafluoroborate) (12)}

To a solution of $1(0.5 \mathrm{~g}, 1.36 \mathrm{mmol}), \mathrm{Ru}(\mathrm{dndp}){ }_{3} \mathrm{Cl}_{2}$ (0.032 g, $0.023 \mathrm{mmol})$ and $\mathrm{LiBF}_{4}(0.172 \mathrm{~g}, 1.82$ $\mathrm{mmol})$ in dry acetonitrile $(7 \mathrm{~mL})$, DIPEA $(0.157 \mathrm{~mL}$, $0.119 \mathrm{~g}, 0.907 \mathrm{mmol}$ ) was added, and the reaction mixture was irradiated $(\lambda=470 \mathrm{~nm})$ for 5 days at room temperature. The solvent was removed under reduced pressure; the residue was dissolved in water $(20 \mathrm{~mL})$ and was washed with chloroform $(3 \times 40 \mathrm{~mL})$. The aqueous phase was concentrated to $10 \mathrm{~mL}$ and purified by column chromatography (Sephadex G10, water). Lyophilisation gave $\mathbf{1 2}(0.17 \mathrm{~g}, 31 \%)$ as a white amorphous solid; $\mathrm{R}_{\mathrm{F}}=0.63$ (silica gel, $n$ butanol/ $n$-propanol/water, $1: 1: 1)$;

${ }^{1} \mathrm{H}$ NMR (400 MHz, $\left.\mathrm{D}_{2} \mathrm{O}\right): \delta=6.75(s, 2 \mathrm{H}, 2 \times \mathrm{OH})$, 6.37 ( $s, 4 \mathrm{H}$, arom.), $4.67\left(m, 4 \mathrm{H}, 2 \times \mathrm{OCH}_{2}\right), 4.29$, $4.18\left(A A^{\prime} B B^{`}, 4 \mathrm{H}\right.$, cyclobutane $\left.\mathrm{H}^{`} \mathrm{~s}\right), 3.76(m, 4 \mathrm{H}, 2 \mathrm{x}$ $\left.\mathrm{CH}_{2} \mathrm{NMe}_{3}{ }^{+}\right), 3.70\left(s, 12 \mathrm{H}, 4 \times \mathrm{OCH}_{3}\right), 3.12(s, 18 \mathrm{H}, 2$ $\mathrm{X} \mathrm{NMe}_{3}^{+}$) ppm;

${ }^{13} \mathrm{C}$ NMR (125 MHz, $\left.\mathrm{CD}_{3} \mathrm{CN}\right): \delta=173.3(2 \times \mathrm{C}=\mathrm{O})$, 148.1 (C, 4 x arom. $\left.C-\mathrm{OCH}_{3}\right), 132.3(\mathrm{C}, 2 \mathrm{x}$ arom. 
C-OH), 131.3 ( $2 \times \mathrm{C}$, arom.), 106.8 (4 x CH, arom.), $65.6\left(2 \times \mathrm{OCH}_{2}\right), 59.2\left(2 \times \mathrm{NCH}_{2}\right), 57.0\left(4 \times \mathrm{OCH}_{3}\right)$, $54.8\left(2 \times \mathrm{NMe}_{3}{ }^{+}\right), 46.6(2 \times \mathrm{CH}, \mathrm{C}-3, \mathrm{C}-4), 42.9$ (2 x CH, C-1, C-2) ppm;

MS (ESI, MeOH): m/z $(\%)=310.2\left(\left[\mathrm{M}-2 \mathrm{BF}_{4}\right]^{2+}\right.$, 100), $707.3\left(\left[\mathrm{M}-\mathrm{BF}_{4}\right]^{+}, 31\right)$;

analysis calcd for $\mathrm{C}_{32} \mathrm{H}_{48} \mathrm{~B}_{2} \mathrm{~F}_{8} \mathrm{~N}_{2} \mathrm{O}_{10} \quad$ (794.35): C 48.39, H 6.09, N 3.53; found: C 48.00, H 6.28, N 3.21.

\section{References}

1- G. Samuelsson, Drugs of Natural Origin: A Textbook of Pharmacognosy, Swedish Pharmaceutical Press, Stockholm, 1999.

2- G.M. Cragg, D.J. Newman, Natural product drug discovery in the next millennium, Pharm. Biol. 2001, 39, 8-17.

3- J.K. Borchardt, The beginnings of drug therapy: Ancient mesopotamian medicine, Drug News Perspect. 2002, 15(3), 187-192.

4- O. Wallach, Terpene und Campher, Veit \& Co, Leipzig, 1909.

5- S. Antonsen, R.B. Østby, Y. Stenstrøm, Naturally Occurring Cyclobutanes: Their Biological Significance and Synthesis, Studies in Natural Products Chemistry, 2018, pp. 1-40.

6- M.E. Daub, H. Jung, B.J. Lee, J. Won, M.H. Baik, T.P. Yoon, Enantioselective [2+2] Cycloadditions of Cinnamate Esters: Generalizing Lewis Acid Catalysis of Triplet Energy Transfer, J. Am. Chem. Soc. 2019, 141(24), 9543-9547.

7- J.D. Hart, L. Burchill, A.J. Day, C.G. Newton, C.J. Sumby, D.M. Huang, J.H. George, VisibleLight Photoredox Catalysis Enables the Biomimetic Synthesis of Nyingchinoids A, B, and D, and Rasumatranin D, Angew. Chem. Int. Ed. 2019, 58(9), 2791-2794.

8- Q. Li, K. Zhao, A. Peuronen, K. Rissanen, D. Enders, Y. Tang, Enantioselective Total Syntheses of (+)-Hippolachnin A, (+)Gracilioether A, (-)-Gracilioether E, and (-)Gracilioether F, J. Am. Chem. Soc. 2018, 140(5), 1937-1944.

9- M. Wang, P. Lu, Catalytic approaches to assemble cyclobutane motifs in natural product synthesis, Org. Chem. Front. 2018, 5(2), 254259.

10-Y. Yuan, J.X. Yang, L.H. Nie, B.L. Li, X.B. Qin, J.W. Wu, S.X. Qiu, Three new kavalactone dimers from Piper methysticum (kava), J. Asian Nat. Prod. Res. 2018, 20(9), 837-843.

11-P. Chandra, V. Bajpai, M. Srivastva, K.B.R. Kumar, B. Kumar, Metabolic profiling of Piper species by direct analysis using real-time mass spectrometry combined with principal component analysis, Anal. Methods 2014, 6(12), 4234-4239.

12-B.R. Lisbet, J.M. Mikkelsen, Combination chemotherapy compositions containing tacrolimus in combination of agents for regulating CYP3A4 or P-glycoprotein level, Lifecycle Pharma A/S, Den. 2007, p. 66pp.

13-R. Muharini, Z. Liu, W. Lin, P. Proksch, New amides from the fruits of Piper retrofractum, Tetrahedron Lett. 2015, 56(19), 2521-2525.

14-L.B. Rankloeve, Pharmaceutical compositions comprising tacrolimus and a CYP3A4 inhibitor, 2006, PCT/DK2006/050025

15-M. Takahashi, M. Ichikawa, S. Aoyagi, C. Kibayashi, Total synthesis of dipiperamide A and revision of stereochemical assignment, Tetrahedron Lett. 2005, 46(1), 57-59.

16-S. Tsukamoto, B.-C. Cha, T. Ohta, Dipiperamides A, B, and C: bisalkaloids from the white pepper Piper nigrum inhibiting CYP3A4 activity, Tetrahedron 2002, 58(9), 1667-1671.

17-S. Tsukamoto, K. Tomise, K. Miyakawa, B.C. Cha, T. Abe, T. Hamada, H. Hirota, T. Ohta, CYP3A4 Inhibitory Activity of New Bisalkaloids, Dipiperamides D and E, and Cognates from White Pepper, Bioorg. Med. Chem. 2002, 10(9), 2981-2985.

18-K. Wei, W. Li, K. Koike, Y. Chen, T. Nikaido, Nigramides A-S, Dimeric Amide Alkaloids from the Roots of Piper nigrum, J. Org. Chem. 2005, 70(4), 1164-1176.

19- S.R. Chintala, J. Fox, Enantioselective synthesis of cyclobutanes via sequential Rh-catalyzed bicyclobutanation/Cu-catalyzed homoconjugate addition and design of mixed-ligand chiral rhodium (II) catalysts for enantioselective transformations of $\alpha$-alkyl- $\alpha$-diazoesters, Abstracts of Papers, 252nd ACS National Meeting \& Exposition, Philadelphia, PA, United States, August 21-25, 2016, 2016, ORGN-40.

20-F. Frebault, N. Maulide, Total Synthesis and Structural Revision of the Piperarborenines: When Photochemistry Meets C-H Activation, Angew. Chem., Int. Ed. 2012, 51(12), 2815-2817.

21-W.R. Gutekunst, P.S. Baran, Total Synthesis and Structural Revision of the Piperarborenines via Sequential Cyclobutane C-H Arylation, J. Am. Chem. Soc. 2011, 133(47), 19076-19079.

22-W.R. Gutekunst, P.S. Baran, Applications of C-H Functionalization Logic to Cyclobutane Synthesis, J. Org. Chem. 2014, 79(6), 2430-2452.

23-J.-L. Hu, L.-W. Feng, L. Wang, Z. Xie, Y. Tang, X. Li, Enantioselective Construction of Cyclobutanes: A New and Concise Approach to the Total Synthesis of (+)-Piperarborenine B, J. Am. Chem. Soc. 2016, 138(40), 13151-13154.

24-F.-P. Lee, Y.-C. Chen, J.-J. Chen, I.-L. Tsai, I.S. Chen, Cyclobutanoid amides from Piper arborescens, Helv. Chim. Acta 2004, 87(2), 463-468.

25- T. Lynch-Colameta, R. Telmesani, A.B. Beeler, Intermolecular synthesis and medicinal chemistry of cinnamate and cinnamide derived cyclobutanes, Abstracts of Papers, 250th ACS 
National Meeting \& Exposition, Boston, MA, United States, August 16-20, 2015, 2015, CHED-292.

26-R.A. Panish, S.R. Chintala, J.M. Fox, MixedLigand Chiral Rhodium(II) Catalyst Enables the Enantioselective Total Synthesis of Piperarborenine B, Angew. Chem., Int. Ed. 2016, 55(16), 4983-4987.

27-Y. Tang, Asymmetric catalytic reactions: Recent use of TOX and SaBOX ligands, Abstracts of Papers, 254th ACS National Meeting \& Exposition, Washington, DC, USA, August 20-24, 2017, 2017, ORGN-258.

28- I.L. Tsai, F.-P. Lee, C.-C. Wu, C.-Y. Duh, T. Ishikawa, J.-J. Chen, Y.-C. Chen, H. Seki, I.S. Chen, New cytotoxic cyclobutanoid amides, a new furanoid lignan and anti-platelet aggregation constituents from Piper arborescens, Planta Med. 2005, 71(6), 535-542.

29-D.P. Bezerra, C. Pessoa, M.O. de Moraes, N. Saker-Neto, E.R. Silveira, L.V. Costa-Lotufo, Overview of the therapeutic potential of piplartine (piperlongumine), Eur. J. Pharm. Sci. 2013, 48(3), 453-463.

30-K. Piska, A. Gunia-Krzyzak, P. Koczurkiewicz, K. Wojcik-Pszczola, E. Pekala, Piperlongumine (piplartine) as a lead compound for anticancer agents - Synthesis and properties of analogues: A mini-review, Eur. J. Med. Chem. 2018, 156, 1320.

31-S. Prasad, A.K. Tyagi, Historical Spice as a Future Drug: Therapeutic Potential of Piperlongumine, Curr. Pharm. Des. 2016, 22(27), 4151-4159.

32-S. Sommerwerk, R. Kluge, D. Ströhl, L. Heller, A.E. Kramell, S. Ogiolda, P. Liebing, R. Csuk, Synthesis, characterization and cytotoxicity of new piplartine dimers, Tetrahedron 2016, 72(11), 1447-1454.

33- J. Wiemann, J. Karasch, A. Loesche, L. Heller, W. Brandt, R. Csuk, Piperlongumine B and analogs are promising and selective inhibitors for acetylcholinesterase, Eur. J. Med. Chem. 2017, 139, 222-231.

34- G. Dominguez, J. Perez-Castells, Alkenes in $[2+2+2]$ Cycloadditions, Chem-Eur. J. 2016, 22(20), 6720-6739.

35- D. Cambie, T. Noel, Solar Photochemistry in Flow, Topics Curr Chem 376(6) (2018).

36- A.A. Aachary, U. Thiyam-Hollander, An update on characterization and bioactivities of sinapic acid derivatives, CRC Press, 2013, pp. 21-38.

37- N. Niciforovic, H. Abramovic, Sinapic Acid and Its Derivatives: Natural Sources and Bioactivity, Compr. Rev. Food Sci. Food Saf. 2014, 13(1), 34-51.

38-V. Boscaro, L. Boffa, A. Binello, G. Amisano, S. Fornasero, G. Cravotto, M. Gallicchio, Antiproliferative, proapoptotic, antioxidant and antimicrobial effects of sinapis nigra 1 . and sinapis alba 1. extracts, Molecules 2018, 23(11), 3004/1-3004/18.

39-J. Dubie, A. Stancik, M. Morra, C. Nindo, Antioxidant extraction from mustard (Brassica juncea) seed meal using high-intensity ultrasound, J. Food Sci. 2013, 78(4-5-6), E542E548.

40-J. Jiang, Y. Wang, T. Xie, H. Rong, A. Li, Y. Fang, Y. Wang, Metabolic characteristics in meal of black rapeseed and yellow-seeded progeny of Brassica napus-Sinapis alba hybrids, Molecules 2015, 20(12), 21204-21213.

41-M. Zhang, C. Zheng, M. Yang, Q. Zhou, W. Li, C. Liu, F. Huang, Primary Metabolites and Polyphenols in Rapeseed (Brassica napus L.) Cultivars in China, J. Am. Oil Chem. Soc. 2019, 96(3), 303-317.

42-Y. Li, J. Li, Q. Su, Y. Liu, Sinapine reduces nonalcoholic fatty liver disease in mice by modulating the composition of the gut microbiota, Food Funct. 2019, 10(6), 3637-3649.

43-Y.-F. Xian, Z. Hu, S.-P. Ip, J.-N. Chen, Z.-R. Su, X.-P. Lai, Z.-X. Lin, Comparison of the antiinflammatory effects of Sinapis alba and Brassica juncea in mouse models of inflammation, Phytomedicine 2018, 50, 196-204.

44-N. Martinovic, N. Poklar Ulrih, H. Abramovic, Sinapic Acid and its Derivatives Increase Oxidative Stability in Different Model Lipid Systems, Eur. J. Lipid Sci. Technol. 2019, 121(4), 1800326.

45-M. Rohit, T. Ashok, R. Vijaykumar,

K. Kashniyal, Molecular docking study of Cassia tora, Brassica campestris and Calotropis procera as acetylcholinesterase inhibitor, Indian J. Pharm. Educ. Res. 2016, 50(1), 116-122.

46-L. von Bao, M. Hirschbrunn, Über das Sinapin, Liebigs Ann. Chem. 1852, 84, 10-32.

47-E. Späth, Die Synthese des Sinapins, Monatsh. Chem. 1920, 41, 271-85.

48-J. Du, T.P. Yoon, Crossed Intermolecular [2+2] Cycloadditions of Acyclic Enones via Visible Light Photocatalysis, J. Am. Chem. Soc. 2009, 131(41), 14604-14605.

49-K.P.S. Zanoni, R.R.C. Vilela, I.D.A. Silva, N.Y.M. Iha, H. Eckert, A.S.S. de Camargo, Photophysical Properties of Ir(III) Complexes Immobilized in MCM-41 via Templated Synthesis, Inorg Chem 2019, 58(8), 4962-4971.

50-M.A. Ischay, M.E. Anzovino, J. Du, T.P. Yoon, Efficient visible light photocatalysis of [2+2] enone cycloadditions, J. Am. Chem. Soc. 2008, 130(39), 12886-12887.

51-J. Gadamer, Über das Sinapin, Ber. Dtsch. chem. Ges. 1897, 30, 2328-30.

52-A.T. Babayan, A.A. Grigoryan, A.N. Grigoryan, Amines and quaternary ammonium compounds. VII. Reaction of haloalkyl-containing tertiary amines and their methiodides with alkali, $\mathrm{Zh}$. Obshch. Khim. 1957, 27, 1827-32. 